\title{
Effect of rapamycin on endometriosis in mice
}

\author{
XU REN ${ }^{1,2}$, YIFENG WANG ${ }^{1}$, GANG XU $^{3}$ and LIBING DAI $^{2}$ \\ ${ }^{1}$ Department of Obstetrics and Gynecology, Zhujiang Hospital, Southern Medical University, Guangzhou, Guangdong 510282; \\ Departments of ${ }^{2}$ Obstetrics and Gynecology and ${ }^{3}$ Endocrinology, Guangzhou Red Cross Hospital, \\ Guangzhou, Guangdong 510120, P.R. China
}

Received February 1, 2015; Accepted March 14, 2016

DOI: $10.3892 /$ etm.2016.3280

\begin{abstract}
The aims of the present study were to investigate the impact of rapamycin (RAPA) on the endometriosis (EMS) lesions in severe combined immunodeficiency (SCID) mice, and to examine the possible mechanism involved in a novel therapy in EMS. Following the successful establishment of an EMS-SCID mouse model, the mice were randomly assigned into the RAPA, control and saline treatment groups. Subsequent to treatment for 2 weeks, the serum hypoxia-inducible factor- $1 \alpha$ (HIF-1 $\alpha$ ) and vascular endothelial growth factor (VEGF) were detected using ELISA. The levels of HIF-1 $\alpha$ and VEGF, as well as the size of EMS lesions, were compared among the three groups. In addition, the HIF-1 $\alpha, \mathrm{VEGF}$ and CD34 protein expression levels, and the microvessel density (MVD) of the lesions were determined by immunohistochemical analysis. Compared with the control and saline groups, the volume of EMS lesions in the RAPA-treated SCID mice was significantly reduced. Furthermore, the serum level and protein expression of VEGF, and the MVD in the lesions of the RAPA-treated group were significantly reduced when compared with the other two groups. These parameters were comparable in the control and saline groups. In conclusion, RAPA may inhibit the growth of endometriotic lesions, most possibly through the inhibition of the expression of VEGF in lesions, thereby inhibiting angiogenesis.
\end{abstract}

\section{Introduction}

Endometriosis (EMS) is a common benign gynecological disorder, which is invasive and prone to metastasis (1). It has been estimated that endometriosis is present in $6-10 \%$ of reproductive age women and in up to 20-40\% of infertile women (2). The mechanism underlying the etiology of EMS

Correspondence to: Professor Yifeng Wang, Department of Obstetrics and Gynecology, Zhujiang Hospital, Southern Medical University, 253 Gongye Avenue, Guangzhou, Guangdong 510282, P.R. China

E-mail: wyf1988@163.com

Key words: rapamycin, endometriosis, hypoxia-inducible factor-1 $\alpha$, vascular endothelial growth factor, microvessel density remains unclear and no efficient treatment is available. Seeking an effective therapeutic approach has been the focus of numerous studies in recent years.

Mammalian target of rapamycin (mTOR) is a serine/threonine kinase that integrates a wide range of signaling pathways, leading to its involvement in divergent physiological processes, including transcription, translation, ribosome biogenesis and apoptosis (3). mTOR has been demonstrated to be a crucial regulator in certain types of malignant cancer $(4,5)$ and has been shown to be tightly associated with the occurrence and development of EMS (6). Rapamycin (RAPA), an inhibitor of mTOR, was found to be involved in the induction of cell apoptosis, suppression of cancer cell proliferation, invasion and angiogenesis in tumors $(7,8)$. In addition, Leconte et al (9) revealed that blocking the mTOR signaling pathway inhibits the formation of deep infiltrating EMS nodules in mice. However, the overall effects of mTOR inhibitors on EMS remain unclear and the possible underlying mechanism is unknown.

Hypoxia-inducible factor- $1 \alpha$ (HIF- $1 \alpha)$, which is a general regulatory factor in the response to hypoxia, regulates the expression of vascular endothelial growth factor (VEGF) and various upstream enzymes, resulting in the regulation of the process of angiogenesis (10). The occurrence and development of angiogenesis in EMS has been closely associated with ischemia and hypoxia of the ectopic endometrium (11-13). In our previous studies, it was demonstrated that HIF-1 $\alpha$ is involved in the process of angiogenesis in EMS $(14,15)$. Furthermore, previous studies reported that mTOR was able to enhance the expression of HIF-1 $\alpha$, thereby promoting the expression of VEGF (16).

In the present study, the effect of RAPA on EMS and its possible mechanism were investigated in order to provide new evidence for a targeted EMS therapy. The study investigated the effect of RAPA on the development of EMS lesions and microvessel density (MVD), as well as the expression of HIF- $1 \alpha$ and VEGF.

\section{Materials and methods}

Animals and reagents. A total of 30 female specific pathogen free (SPF) CB17 severe combined immunodeficiency (SCID) mice (weight, $17.02 \pm 0.75 \mathrm{~g}$; age, $6-7$ weeks) were purchased from Beijing Vital River Laboratory Animal Technology Co., Ltd. (Beijing, China). RAPA was purchased from LC Laboratories 
(Woburn, MA, USA). Mouse HIF-1 $\alpha$ and VEGF ELISA kits (cat. nos. CSB-E08541m and CSB-E04756m, respectively) were obtained from Sino-American Biotechnology Co., Ltd. (Wuhan, China). Mouse anti-human HIF-1 $\alpha$ polyclonal antibody (1:100; cat. no. BM0912) was purchased from Wuhan Boster Biological Technology, Ltd. (Wuhan, China). Mouse anti-human VEGF (1:200; cat. no. RB-9031) and CD34 (1:100; cat. no. MS-363) monoclonal antibodies, streptomycin avidin-peroxidase (SP) immunochemistry (cat. no. Kit-9701) and DAB (cat. no. Kit-0017) kits were from Maxim Biotech, Inc. (Fuzhou, China). Lidocaine and pentobarbital were obtained from Sigma-Aldrich (St. Louis, MO, USA). Formalin, paraffin and bovine serum albumin (BSA) Sangon Biotech, Co., Ltd. (Shanghai, China). Citric acid and sodium citrate were purchased from Guangzhou Chemical Reagent Factory (Guangzhou, China).

Animal model. Tissue samples were acquired from 15 female patients during laparoscopic resection of EMS at the Departments of Obstetrics and Gynecology at the Guangzhou Red Cross Hospital (Guangzhou, China) between January 2014 and February 2014. The average age of the participants was $32 \pm 8$ years. Informed consent was obtained from all the participants prior to the procedure. The samples were confirmed to be eutopic secretory endometrial tissues by a pathologist who was blinded to the research design. The tissues were sectioned into $1-\mathrm{mm}^{3}$ fragments and stored temporarily in a cold sterile bottle with an appropriate volume of Dulbecco's modified Eagle's medium (GE Healthcare Life Sciences, Logan, UT, USA) for $<1 \mathrm{~h}$. This study was approved by the Ethics Committee of Zhujiang Hospital of Southern Medical University.

In order to establish an EMS-SCID animal model, $2 \mathrm{ml}$ of the tissue suspension obtained from the EMS patients was injected into the abdominal cavity of 30 female SPF CB17 SCID mice within $60 \mathrm{~min}$ after the harvest of the tissue sample. The mice were maintained in cages (5 mice/cage), under SPF conditions at $22-24^{\circ} \mathrm{C}$ and $50-70 \%$ relative humidity, and under a $12-\mathrm{h}$ light/dark cycle. All mice were fed a normal laboratory diet. After 4 weeks, the EMS-SCID mouse model was successfully established, as confirmed by endometrial tissue investigation during laparotomy, which showed a cyst on the internal abdominal wall. For laparotomy, $0.1 \mathrm{ml} 2 \%$ lidocaine was injected subcutaneously. The volume of EMS lesions prior to treatment (V1) were measured, according to the method of Katsuki (17), the 30 EMS-SCID mice were then randomly assigned into three groups ( $\mathrm{n}=10$ each), as follows: i) RAPA group, in which the lesions were injected with $250 \mu \mathrm{g}$ /mouse RAPA once per week; ii) control group, receiving no treatment; and iii) saline group, receiving lesions were injected with $0.25 \mathrm{ml} /$ mouse saline once per week. There was no difference in the V1 among the three groups $(\mathrm{P}>0.05)$. At 2 weeks after the initiation of treatment (V2), blood samples were collected from the orbit of the mice following removal of the eyeball. Subsequently, all animals were sacrificed with an overdose of pentobarbital $(100 \mathrm{mg} / \mathrm{kg})$, and the volume of EMS lesions following treatment was measured using the previously mentioned method.

ELISA. Blood samples were obtained from the orbit of the mice prior to sacrifice. Serum was collected by centrifugation
Table I. Endometriotic lesion volume in the mice (mean \pm standard deviation; $\mathrm{n}=10$ mice/group).

\begin{tabular}{lcc}
\hline Group & $\mathrm{V} 1\left(\mathrm{~mm}^{3}\right)$ & $\mathrm{V} 2\left(\mathrm{~mm}^{3}\right)$ \\
\hline RAPA & $230.85 \pm 13.74$ & $51.25 \pm 9.31^{\mathrm{a}, \mathrm{b}}$ \\
Control & $219.56 \pm 14.79$ & $229.44 \pm 10.01$ \\
Saline & $231.34 \pm 13.41$ & $210.71 \pm 10.96$ \\
\hline
\end{tabular}

${ }^{\mathrm{a}} \mathrm{P}<0.05$ vs. V1 in the same group; ${ }^{\mathrm{b}} \mathrm{P}<0.05$ vs. $\mathrm{V} 2$ in the control and saline groups. RAPA, rapamycin; V1, lesion volume prior to treatment; V2, lesion volume following treatment.

Table II. Serum levels of HIF-1 $\alpha$ and VEGF in the mice (mean \pm standard deviation; $\mathrm{n}=10$ mice/group).

\begin{tabular}{lcc}
\hline Group & HIF- $1 \alpha(\mathrm{pg} / \mathrm{ml})$ & VEGF $(\mathrm{pg} / \mathrm{ml})$ \\
\hline RAPA & $1.575 \pm 0.290$ & $0.128 \pm 0.030$ \\
Control & $1.668 \pm 0.223$ & $0.169 \pm 0.029$ \\
Saline & $1.660 \pm 0.205$ & $0.162 \pm 0.030$ \\
\hline
\end{tabular}

RAPA, rapamycin; HIF-1 $\alpha$, hypoxia-inducible factor-1 $\alpha$; VEGF, vascular endothelial growth factor.

for $15 \mathrm{~min}$ at $1,000 \mathrm{x}$. Serum HIF-1 $\alpha$ and VEGF protein expression levels were measured by ELISAs, according to the manufacturer's protocols. The serum concentrations of HIF-1 $\alpha$ and VEGF were quantified using a standard curve constructed according to the absorbance and concentrations of standard samples.

Immunohistochemistry. The protein expression levels of HIF-1 $\alpha$, VEGF and CD34 in the EMS lesions of the mice were measured by immunohistochemical analysis. Briefly, the tissue samples of endometriotic foci obtained after sacrifice were fixed in $10 \%$ formalin and then embedded in paraffin. The tissues were cut into $\sim 4-\mu \mathrm{m}$ sections. Antigen repair was conducted by microwave heating in $0.1 \%$ citrate buffer $(0.4 \mathrm{~g}$ citric acid dissolved in $3 \mathrm{~g}$ sodium citrate in $1 \mathrm{~L}$ deionized water). After blocking with 5\% BSA at room temperature for $20 \mathrm{~min}$, the slides were incubated overnight at $4^{\circ} \mathrm{C}$ with mouse anti-HIF-1 $\alpha$ (1:100), anti-VEGF (1:200) or anti-CD34 (1:100) antibodies. Protein expression was detected according to the protocols of the SP and DAB kits. Brown staining in the cytoplasm, as observed under a light microscope (Olympus BX51; Olympus Corporation, Tokyo, Japan), was indicative of HIF-1 $\alpha$, VEGF or CD34 protein expression.

The results of immunohistochemistry were reviewed independently by two senior pathologists blinded to the outcome of the tumors. Semi-quantitative analysis of HIF-1 $\alpha$, VEGF and CD34 expression levels was performed by consensus and comprised both the intensity of staining (negative as 0 , light yellow as 1 , brown as 2 , tan as 3 ) for each cell and the extent of staining (ratio of positive cells/counted cells; 1 for $25 \%$, 2 for $25-50 \%, 3$ for $51-75 \%$ and 4 for $75 \%$ ) for each random field. The scores for the intensity and extent of staining were 
Table III. Intensity of immunohistochemical staining for $\mathrm{HIF}-1 \alpha$ in endometriotic lesions ( $\mathrm{n}=10 \mathrm{mice} / \mathrm{group})$.

\begin{tabular}{lllll}
\hline & \multicolumn{4}{c}{ HIF-1 $\alpha$} \\
\cline { 2 - 5 } Group & - & + & ++ & +++ \\
\hline RAPA & 0 & 4 & 2 & 4 \\
Control & 0 & 4 & 3 & 3 \\
Saline & 0 & 3 & 4 & 3 \\
\hline
\end{tabular}

RAPA, rapamycin; HIF- $1 \alpha$, hypoxia-inducible factor- $1 \alpha$.

Table IV. Intensity of immunohistochemical staining for VEGF in endometriotic lesions ( $\mathrm{n}=10 \mathrm{mice} /$ group).

\begin{tabular}{lllll}
\hline & \multicolumn{4}{c}{ VEGF } \\
\cline { 2 - 5 } Group & - & + & ++ & +++ \\
\hline RAPA & 7 & 3 & 0 & 0 \\
Control & 0 & 5 & 3 & 2 \\
Saline & 0 & 4 & 5 & 1 \\
\hline
\end{tabular}

RAPA, rapamycin; VEGF, vascular endothelial growth factor.

Table V. MVD in endometriotic lesions in mice (mean \pm standard deviation; $\mathrm{n}=10$ mice/group).

\begin{tabular}{lc}
\hline Group & MVD \\
\hline RAPA & $5.38 \pm 0.18^{\mathrm{a}}$ \\
Control & $35.54 \pm 1.19$ \\
Saline & $36.26 \pm 1.28$ \\
\hline
\end{tabular}

${ }^{\mathrm{a}} \mathrm{P}<0.05$ vs. control and saline groups. RAPA, rapamycin; MVD, microvessel density.

multiplied to give a weighted score for each case (maximum possible score, 12). For statistical analysis, the weighted scores were grouped into four categories, wherein a score of 0 was considered negative, scores of 1-4 (+) were regarded as low positive expression levels, and scores of 5-8 (++) and 9-12 $(+++)$ were regarded as high positive expression levels.

MVD. The MVD was assessed as previously described (18). Briefly, under a light microscope (Olympus Corporation), the immunostained section was scanned at low magnification (x40) and three areas with the highest density of microvessels were identified as 'hotspots'. All the vessels in these 'hotspot' areas were then counted at high magnification (x200), and each slide was examined individually by two pathologists blinded to the research design.

Statistical analysis. The results are expressed as the mean \pm standard deviation. Statistical analysis was

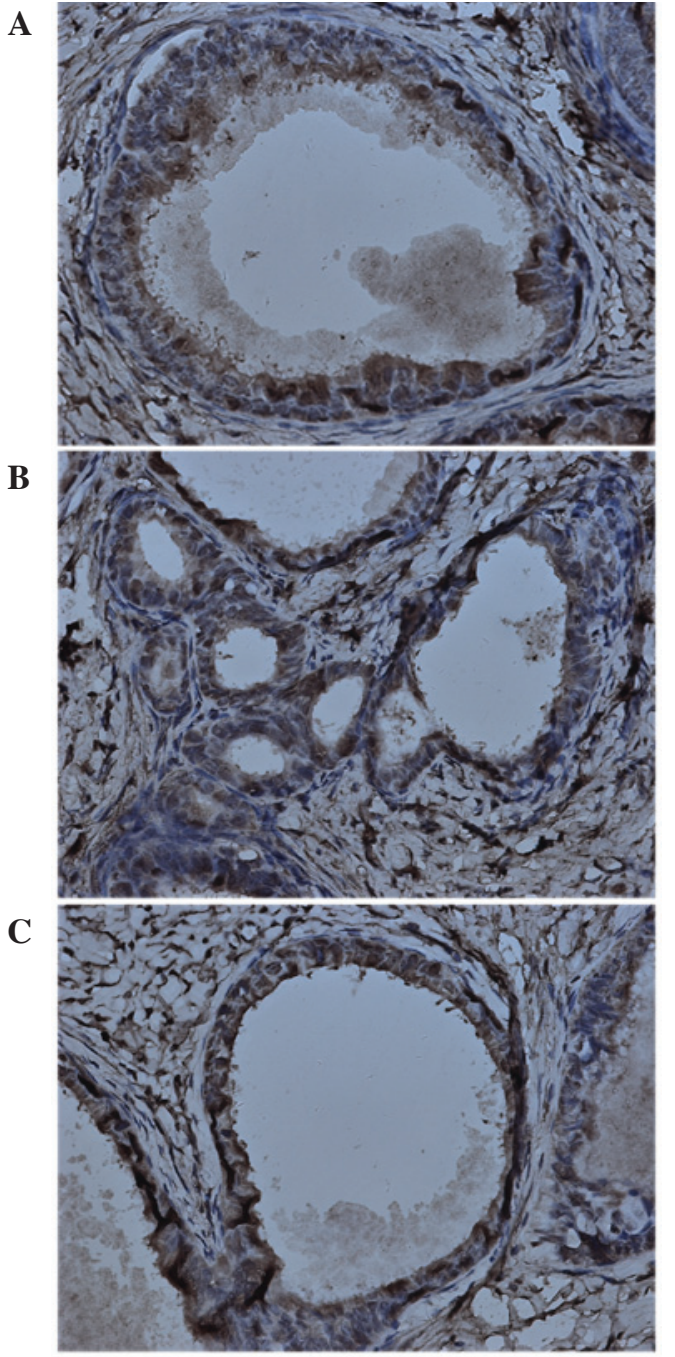

Figure 1. Representative image of immunohistochemical analysis of hypoxia-inducible factor- $1 \alpha$ in endometriotic lesions of (A) control, (B) saline and $(C)$ rapamycin-treated groups. Staining was performed with the streptomycin avidin-peroxidase method. Magnification, x400.

conducted with SPSS 19.0 software (IBM SPSS, Armonk, NY, USA). Comparisons between groups were performed using one-way analysis of variance test. All the numerical data were tested for homogeneity and distribution. Ordinal data were tested by Wilcoxon signed-rank test. A P-value of $<0.05$ was considered to indicate a statistically significant difference.

\section{Results}

Effect of RAPA on the growth of endometriotic lesions in mice. In order to observe the effect of RAPA on the endometriotic lesions in mice, the volume of lesions was measured prior to treatment (V1) and after 2 weeks of treatment (V2). As shown in Table I, the V2 of the control and saline groups were comparable with V1 of all the groups. However, the V2 of the RAPA group was significantly decreased when compared with its corresponding V1, as well as compared with V2 of the control and saline groups $(\mathrm{P}<0.05)$. These findings indicated that RAPA significantly suppressed the growth of the endometriosis lesions. 
A

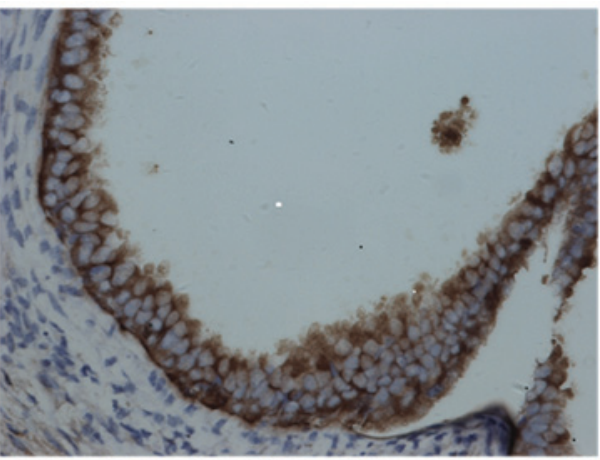

B

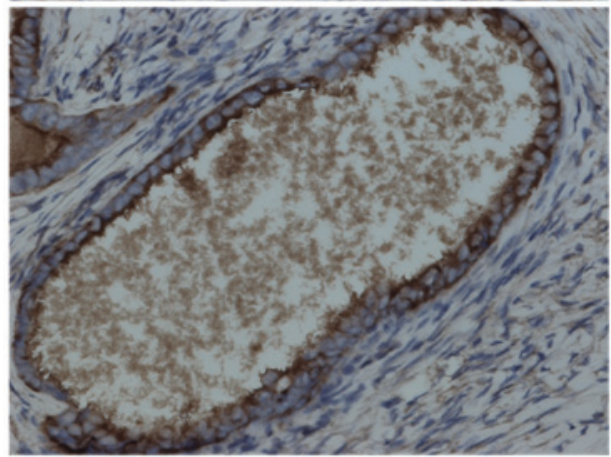

C

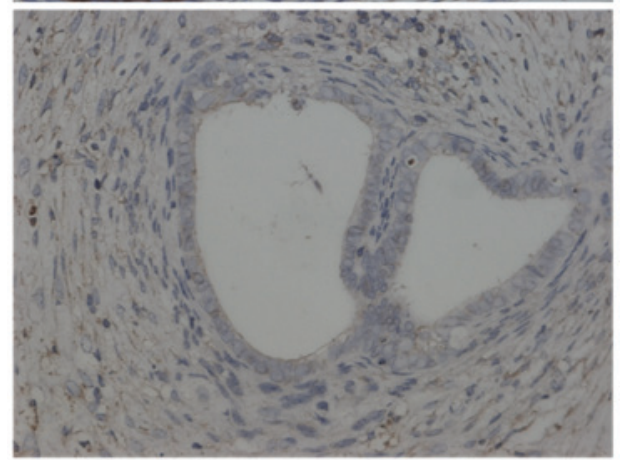

Figure 2. Representative image of immunohistochemical analysis of vascular endothelial growth factor in endometriotic lesions of (A) control, (B) saline and $(\mathrm{C})$ rapamycin-treated groups. Staining was performed with the streptomycin avidin-peroxidase method. Magnification, x400.

Effect of RAPA on the serum levels of HIF-1 $\alpha$ and VEGF. In order to investigate the effect of RAPA on the expression of HIF- $1 \alpha$ and VEGF, the serum levels of these factors were determined by ELISA. As shown in Table II, the serum levels of HIF-1 $\alpha$ and VEGF in the saline groups were comparable with those in the corresponding control group levels. Notably, there was no statistically significant difference in the serum HIF- $1 \alpha$ levels among the RAPA, control and saline groups. However, treatment with RAPA markedly reduced the serum levels of VEGF, when compared with those in the control and saline groups $(\mathrm{P}<0.05)$.

Effect of RAPA on the expression of HIF-1 $\alpha$ and VEGF in endometriotic lesions. In order to confirm the effect of RAPA on the location and expression of HIF-1 $\alpha$ and VEGF, their expression in endometriotic lesions was determined by immunohistochemical staining. The results showed that HIF-1 $\alpha$ and VEGF were mainly present in the cytoplasm of glandular epithelium. In addition, weak immunoreactivity for HIF-1 $\alpha$ and VEGF was observed in the ectopic endometrial stromal cells in the control group (Figs. 1 and 2). The results
A

B
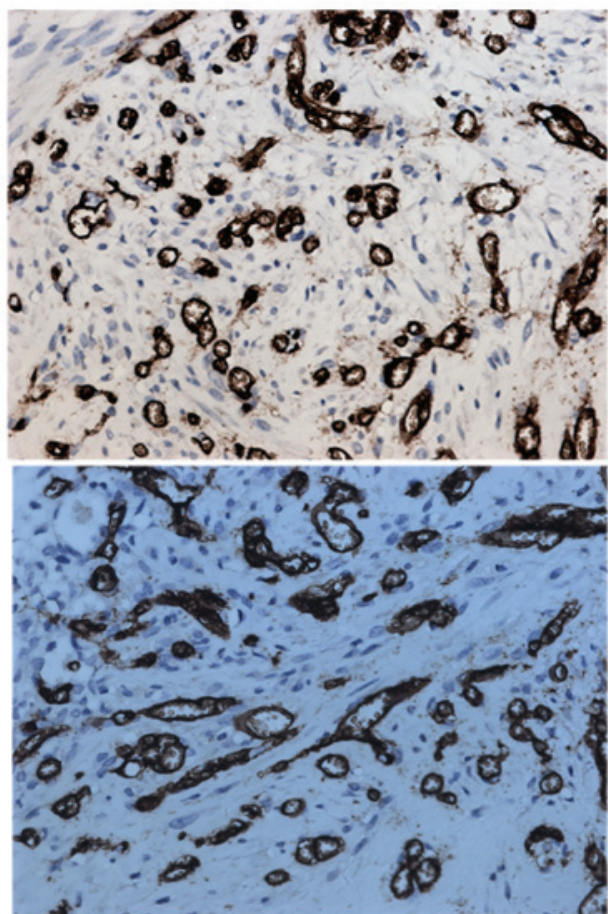

C

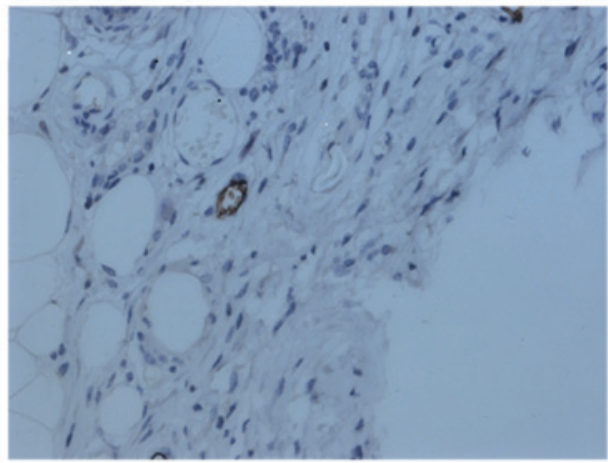

Figure 3. Representative images of immunohistochemical analysis of CD34 in endometriotic lesions of the (A) control, (B) saline and (C) rapamycin-treated groups. Staining was performed with the streptomycin avidin-peroxidase method. Magnification, x400.

of the staining intensity are summarized in Tables III and IV. There was no significant difference in the staining for HIF-1 $\alpha$ among the RAPA, control and saline groups. However, the VEGF expression in the RAPA group was significantly lower compared with that of the control and saline groups.

Effect of RAPA on MVD in entometriotic lesions. As a marker of endothelial cells, CD34 was assayed by immunohistochemistry in order to evaluate the effect of RAPA on MVD in endometriotic lesions. As shown in Table V, the MVD in the saline group was comparable with that in the control group (Table V). As expected, treatment with RAPA significantly reduced the MVD when compared with that the in control and saline groups. In addition, as shown in Fig. 3, positive staining of CD34 was observed in the endothelial cells, which were comma, stripe or tube-shaped with clear lumens. The incidence of angiogenesis was also significantly suppressed in the RAPA-treated group compared with that in the control group (Fig. 3). Therefore, these findings indicated that RAPA inhibited endothelial cell proliferation and the resulting angiogenesis. 


\section{Discussion}

As a common gynecologic disease, the incidence of EMS has been increasing in recent years (2). The implantation of ectopic endometrium, the resultant infertility and repeated abdominal pain greatly affect EMS patients $(2,19)$. A previous study demonstrated that angiogenesis serves an important role not only in the development and repair of normal endometrium, but also in the occurrence and development of EMS (20). Excessive angiogenesis may be critical for the ectopic implantation and growth of the endometrium, which results in EMS $(21,22)$. In the present study, we hypothesized that the effective inhibition of angiogenesis may block the occurrence and development of EMS.

HIF-1, a heterodimer composed of the HIF-1 $\alpha$ and HIF-1 $\beta$ subunits, is a transcription factor that is widely found in the tissues of mammals under hypoxic or anoxic stress (23). HIF-1 $\alpha$ is the unique oxygen-regulated subunit and determines the activity of HIF-1. Furthermore, it is a hypoxia inducible global regulator and contributes to the transcriptional regulation of the expression of VEGF and certain enzymes associated with glycolysis (24-26). Additionally, HIF-1 $\alpha$ is the regulator directly involved in the entire process of angiogenesis (10). VEGF, also known as vascular permeability factor, is an important proangiogenic factor, which selectively binds VEGF receptor in endothelial cells, serving a proangiogenic role (27). Angiogenesis during EMS has been found to be closely associated with hypoxia in the endometrium (28). As a regulator of proangiogenic factors, particularly of VEGF, HIF- $1 \alpha$ serves a critical role in the angiogenesis process in EMS (28). Our previous study demonstrated that HIF-1 $\alpha$ functionally contributes to angiogenesis during EMS (15).

mTOR, an atypical serine/threonine kinase, is important for cell growth and is involved in a wide variety of physiological functions. This kinase also regulates the expression of proangiogenic factors (such as VEGF) at the transcriptional and translational levels. An abnormal mTOR signaling pathway has been identified in various types of malignant neoplasm, while the aberrant activation of mTOR has been confirmed to mediate angiogenesis and contribute to neoplasm development (29). Based on these functions, the mTOR signaling pathway has been a novel target in the treatment of neoplasm and mTOR inhibitors have been identified as the third generation of antiangiogenic pharmaceuticals (30). RAPA is the first identified specific inhibitor of mTOR. A previous study has demonstrated that RAPA is able to significantly inhibit the growth of cell lines derived from various tumors, including breast, lung, colorectal and renal carcinoma (31). In addition, RAPA has an anti-angiogenesis activity, and this effect is most probably due to at least the following two mechanisms: i) Direct inhibition of the proliferation of endothelial cells mediated by VEGF; and ii) inhibition of the proliferative effect of HIF-1 on endothelial cells. Hudson et al (32) has shown that RAPA inhibits the expression levels of VEGF and platelet-derived growth factor through the downregulation of HIF-1 $\alpha$ expression, leading to suppression of new vascular occurrence in prostate cancer. Another study found that, in multiple myeloma, RAPA reduced VEGF receptor expression and therefore inhibited the endothelial cell differentiation and vascular sprouting, reducing angiogenesis in multiple myeloma (33). Laschke et al (34) also demonstrated that RAPA attenuated ectopic endometrium in vivo through inhibition of angiogenesis and cell proliferation.

In the present study, RAPA was shown to significantly attenuate the development of endometriotic lesions, as well as to inhibit MVD and the expression of VEGF in endometriotic lesions. However, RAPA had no significant effect on HIF-1 $\alpha$ expression in the lesions, which may be due to the direct inhibitory role of RAPA in the VEGF expression, supported by presence of various mTOR-independent pathways to regulate HIF-1 $\alpha$ expression in EMS. Although the role of mTOR in the expression of HIF-1 $\alpha$ remains controversial, it is widely agreed that the ultimate effect of the complicated signaling pathways regulated by hypoxia determines the expression of HIF-1 $\alpha$.

In conclusion, the present study observed the effect of RAPA on the development of endometriotic lesions and investigated the mechanism of EMS to provide new evidence for the treatment of the disease with RAPA. mTOR inhibitors, represented by RAPA in the current study, may be potentially used in targeted therapy for EMS. However, the clinical efficacy, side effects and the use of mTOR inhibitors in combination with other targeted pharmaceuticals require further evaluation. Biomarkers for the clinical efficacy of mTOR inhibitors also require further investigation.

\section{Acknowledgements}

The present study was funded by Guangzhou Medical Science and Technology Project 2013 (grant no. 2013A011030016) and the Guangdong Province Science and Technology Plan Projects 2015 (grant no. 20140212).

\section{References}

1. Zhan L, Wang W, Zhang Y, Song E, Fan Y and Wei B: Hypoxia-inducible factor-1alpha: A promising therapeutic target in endometriosis. Biochimie 123: 130-137, 2016.

2. Khan KN, Kitajima M, Fujishita A, Hiraki K, Matsumoto A, Nakashima $M$ and Masuzaki H: Pelvic pain in women with ovarian endometrioma is mostly associated with coexisting peritoneal lesions. Hum Reprod 28: 109-118, 2013.

3. Huynh H: Molecularly targeted therapy in hepatocellular carcinoma. Biochem Pharmacol 80: 550-560, 2010.

4. Olivares-Reyes JA,Arellano-Plancarte Aand Castillo-HernandezJR: Angiotensin II and the development of insulin resistance: Implications for diabetes. Mol Cell Endocrinol 302: 128-139, 2009.

5. Makker A, Goel MM, Das V and Aqarwal A: PI3K-Akt-mTOR and MAPK signaling pathways in polycystic ovarian syndrome, uterine leiomyomas and endometriosis: An update. Gnnecol Endocrinol 28: 175-81, 2012.

6. McKinnon BD, Kocbek V, Nirgianakis K, Bersinger NA and Mueller MD: Kinase signalling pathways in endometriosis: Potential targets for non-hormonal therapeutics. Hum Reprod Update: Jan 5, 2016 (Epub ahead of print).

7. Ashworth RE and Wu J: Mammalian target of rapamycin inhibition in hepatocellular carcinoma. World J Hepatol 6: 776-782, 2014.

8. Beck JT, Ismail A and Tolomeo C: Targeting the phosphatidylinositol 3-kinase (PI3K)/AKT/mammalian target of rapamycin (mTOR) pathway: An emerging treatment strategy for squamous cell lung carcinoma. Cancer Treat Rev 40: 980-989, 2014.

9. Leconte M, Nicco C, Ngô C, Chéreau C, Chouzenoux S, Marut W, Guibourdenche J, Arkwright S, Weill B, Chapron C, et al: The mTOR/AKT inhibitor temsirolimus prevents deep infiltrating endometriosis in mice. Am J Pathol 179: 880-889, 2011.

10. Pugh CW and Ratcliffe PJ: Regulation of angiogenesis by hypoxia: Role of the HIF system. Nat Med 9: 677-684, 2003. 
11. Lu Z, Zhang W, Jiang S, Zou J and Li Y: Effect of oxygen tensions on the proliferation and angiogenesis of endometriosis heterograft in severe combined immunodeficiency mice. Fertil Steril 101: 568-576, 2014

12. Lu Z, Zhang W, Jiang S, Zou J and Li Y: Effect of lesion location on endometriotic adhesion and angiogenesis in SCID mice. Arch Gynecol Obstet 289: 823-830, 2014.

13. Sharkey AM, Day K, McPherson A, Malik S, Licence D, Smith SK and Charnock-Jones DS: Vascular endothelial growth factor expression in human endometrium is regulated by hypoxia. J Clin Endocrinol Metab 85: 402-409, 2000.

14. Ren X, He YL, Pan SL and Peng DX: Expression of hypoxia-inducible factor-1alpha in endometriosis. Nan Fang Yi Ke Da Xue Xue Bao 27: 538-540, 2007 (In Chinese).

15. Ren X, He Y and Peng D: The expression of hypoxia-inducible factor- $1 \alpha$ and the relationship with microvessel density in endometriosis. Guangdong yi xue 28: 229-231, 2007 (In Chinese).

16. Martin KA and Blenis J: Coordinate regulation of translation by the PI 3-kinase and mTOR pathways. Adv Cancer Res 86: 1-39, 2002.

17. Katsuki Y, Takano Y, Futamura Y, Shibutani Y, Aoki D, Udagawa Y and Nozawa S: Effects of dienogest, a synthetic steroid, on experimental endometriosis in rats. Eur J Endocrinol 138: 216-226, 1998.

18. Weidner N: Current pathologic methods for measuring intratumoral microvessel density with in breast carcinoma and other solid tumors. Breast Cancer Res Treat 36: 169-180, 1995.

19. Berkes E, Bokor A and Rigó J Jr.: Current treatment of endometriosis with laparoscopic surgery. Orv Hetil 151: 1137-1144, 2010 (In Hungarian).

20. Young VJ, Ahmad SF, Brown JK, Duncan WC and Horne AW: Peritoneal VEGF-A expression is regulated by TGF- $\beta 1$ through an ID1 pathway in women with endometriosis. Sci Rep 5: 16859, 2015

21. Fujimoto J, Sakaguchi H, Hirose R, Wen $H$ and Tamaya T: Angiogenesis in endometriosis and angiogenic factors. Gynecol Obstet Invest 48 (Suppl 1): 14-20, 1999.

22. Soysal D, Kizildag S, Saatli B, Posaci C, Soysal S, Koyuncuoglu M and Dogan O: A novel angiogenesis inhibitor bevacizumab induces apoptosis in the rat endometriosis model. Balkan J Med Genet 17: 73-80, 2015

23. Uchida T, Rossignol F, Matthay MA, Mounier R, Couette S, Clottes E and Clerici C: Prolonged hypoxia differentially regulates hypoxia-inducible factor (HIF)-1alpha and HIF-2alpha expression in lung epithelial cells: Implication of natural antisense HIF-1alpha. J Biol Chem 279: 14871-14878, 2004.
24. Jian-Lin Z, Hong-Song F, Hao P, Shuang D, Shen C, Jian-Ping L, Bo Q, Jin-Qing W and Feng L: The relationship between HIF-2 $\alpha$ and VEGF with radiographic severity in the primary osteoarthritic knee. Yonsei Med J 57: 735-740, 2016.

25. Bae WY, Choi JS, Kim JE and Jeong JW: Cinnamic aldehyde suppresses hypoxia-induced angiogenesis via inhibition of hypoxia-inducible factor-1 $\alpha$ expression during tumor progression. Biochem Pharmacol 98: 41-50, 2015.

26. Remels AH, Gosker HR, Verhees KJ, Langen RC and Schols AM: TNF- $\alpha$-induced NF- $\kappa$ B activation stimulates skeletal muscle glycolytic metabolism through activation of HIF-1 $\alpha$. Endocrinology 156: 1770-1781, 2015.

27. Shibuya M: Vascular endothelial growth factor and its receptor system: Physiological functions in angiogenesis and pathological roles in various diseases. J Biochem 153: 13-19, 2013.

28. Machado-Linde F, Pelegrin P, Sanchez-Ferrer ML, Leon J, Cascales P and Parrilla JJ: 2-methoxyestradiol in the pathophysiology of endometriosis: Focus on angiogenesis and therapeutic potential. Reprod Sci 19: 1018-1029, 2012.

29. Dufour M, Dormond-Meuwly A, Demartines N and Dormond O: Tarageting the mammalian target of rapamycin (mTOR) in cancer therapy: Lessions from past and future perspectives. Cancers (Basel) 3: 2478-2500, 2011.

30. Ganjoo K and Jacobs C: Antiangiogenesis agents in the treatment of soft tissue sarcomas. Cancer 116: 1177-1183, 2010.

31. Sarbassov DD, Guertin DA, Ali SM and Sabatini DM: Phosphorylation and regulation of AKT/PKB by the rictor-mTOR complex. Science 307: 1098-1101, 2005

32. Hudson CC,Liu M,Chiang GG, Otterness DM,Loomis DC,KaperF, Giaccia AJ and Abraham RT: Regulation of hypoxia-inducible factorl alpha expression and function by the mammalian target of rapamycin. Mol Cell Biol 22: 7004-7014, 2002.

33. Mirsgahi P, Toprak SK, Faussat AM, Dubrulle S, Marie JP, Soria C, Soria J and Mirshahi M: Malignant hematopoietic cells induce an increased expression of VEGFR-1 and VEGFR-3 on bone marrow endothelial cells via AKT and mTOR signalling pathways. Biochem Biophys Res Commun 349: 1003-1010, 2006.

34. Laschke MW, Elitzsch A, Scheuer C, Holstein JH, Vollmar B and Menger MD: Rapamycin induces regression of endometriotic lesions by inhibiting neovascularization and cell proliferation. $\mathrm{Br}$ J Pharmacol 149: 137-144, 2006. 